\title{
EL TRIAGE HOSPITALARIO EN LOS SERVICIOS DE EMERGENCIA
}

\author{
HOSPITAL TRIAGE IN EMERGENCY SERVICES \\ Rolando Vásquez-Alva ${ }^{1,3, a, b}$, Consuelo Luna-Muñoz ${ }^{2,4, c}$, Cleto M. Ramos-Garay ${ }^{1, a}$
}

\begin{abstract}
RESUMEN
EI Triage hospitalario, es una parte fundamental de la gestión clínica en emergencia cuando la demanda excede la disponibilidad de recursos humanos y técnicos. El Triage de emergencia es un sistema que ofrece un método para asignar prioridad clínica en situaciones de emergencia. No está diseñado para juzgar si los pacientes están adecuadamente diagnosticados en el marco de la emergencia, sino para asegurarse de que aquellos que necesitan atención urgente la reciban adecuada y oportunamente.

Mediante la implementación del triage en las emergencias se ha producido una mejora en la calidad asistencial, ya que se atiende de una manera adecuada y en el tiempo necesario a aquellas situaciones patológicas que hacen necesaria una asistencia rápida.

EITriage en los servicios de emergencia ha sido evaluado en muchos trabajos de investigación donde concluyen que es una necesidad su creación, desarrollo y cambio según las exigencias de una mejor atención e incremento de la demanda en emergencia. Este último aspecto ha sido el determinante más importante a nivel mundial en el desarrollo y creación de diferentes escalas de triage para atender las emergencias de manera adecuada y en el tiempo necesario. En el Perú en general y en Lima en particular, no existe un desarrollo sistemático de los conceptos y de la implementación uniforme del triage en los servicios de emergencia hospitalarios. Es necesario conocer los conceptos que rodean al triage de los servicios de emergencia hospitalarios y en función a ello establecer las normativas, por los entes rectores en salud, necesarias para mejorar la atención inicial y de calidad en emergencia.
\end{abstract}

Palabras clave: Triage; Triage hospitalario; Emergencia. (fuente: DeCS BIREME)

\begin{abstract}
Hospital triage is a fundamental part of clinical management in emergency, when the demand exceeds the availability of human and technical resources. The Emergency Triage is a system that offers a method to assign clinical priority in emergency situations. It is not designed to judge whether patients are adequately diagnosed in the emergency, but to ensure that those who need urgent care receive it properly and in a timely manner.

Through the implementation of triage in emergencies, there has been an improvement in the quality of care, since it is attended in an appropriate manner and in the time necessary to those pathological situations that require rapid assistance.

Triage in emergency services has been evaluated in many research projects where they conclude that it is necessary to create, develop and change it according to the demands of better care and increased demand in emergency. This last aspect has been the most important determinant worldwide in the development and creation of different triage scales to deal with emergencies adequately and in the necessary time. In Peru in general and in Lima in particular, there is no systematic development of concepts and uniform implementation of triage in hospital emergency services. It is therefore necessary to know the concepts surrounding the triage of hospital emergency services and in accordance with this, establish the regulations, by the governing bodies in health, necessary to improve initial care and emergency quality.
\end{abstract}

Key words: Triage; Hospital triage; Emergency. (source: MeSH NLM)

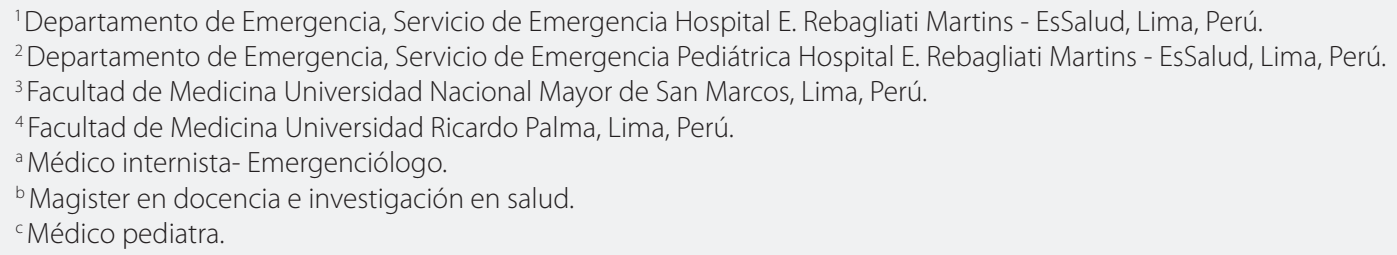

'Departamento de Emergencia, Servicio de Emergencia Hospital E. Rebagliati Martins - EsSalud, Lima, Perú.

²Departamento de Emergencia, Servicio de Emergencia Pediátrica Hospital E. Rebagliati Martins - EsSalud, Lima, Perú.

${ }^{3}$ Facultad de Medicina Universidad Nacional Mayor de San Marcos, Lima, Perú.

${ }^{4}$ Facultad de Medicina Universidad Ricardo Palma, Lima, Perú.

${ }^{a}$ Médico internista- Emergenciólogo.

${ }^{\mathrm{b}}$ Magister en docencia e investigación en salud.

'Médico pediatra.

Citar como: Rolando Vásquez-Alva, Consuelo Luna-Muñoz, Cleto M. Ramos-Garay. El triage hospitalario en los servicios de emergencia. [Artículo de Revisión].2019;19(1):90-100. (Enero 2019). DOI 10.25176/RFMH.v19.n1.1797 


\section{INTRODUCCIÓN}

Los conceptos sobre las situaciones que actualmente conocemos como emergencias individuales o colectivas tienen un tiempo de desarrollo relativamente corto, tanto en la escena mundial como local. En los últimos 40 años, la velocidad de desarrollo del enfoque sobre emergencias primero se ha ido incrementando, para llegar a ser vertiginosa después. La Medicina de Emergencia es una especialidad joven, bien establecida y madura, pero solo en un número relativamente pequeño de países'.

Sin embargo, las emergencias y desastres son tan antiguas como el hombre mismo y hacen su aparición desde que existe el hombre, ya sea con lesiones o enfermedades agudas que necesitan atención en forma inmediata, en especial las que amenazan la vida o la integridad del ser humano².

La situación actual de los sistemas de salud de nuestro país y del mundo ha sufrido cambios. La transición demográfica y la epidemiológica traducen el incremento de la esperanza de vida, pero también de las enfermedades crónicas y pluripatología. Ya desde el Censo de 1993, la pirámide por edad presentó una reducción relativa en las edades menores y un leve aumento en las edades intermedias. La edad mediana de la población aumentó de 19 a 22 años durante el periodo intercensal 1981 - 1993, y al 2017 la transformación de la pirámide poblacional de una base ancha y vértice angosto a una con una base más reducida y un ensanchamiento progresivo en los centros refleja un número menor de nacimientos y mayor población en edad activa, figura que tiende a ser rectangular, lo que demuestra que el Perú se encuentra en una etapa de transición hacia el envejecimiento demográfico y de un notable aumento de la demanda de los servicios de emergencia ${ }^{3}$.

Diversos trabajos mencionan el impacto del envejecimiento sobre la casuística de un hospital y sus diferentes servicios por lo que se plantea la necesidad de adaptar los recursos hospitalarios a los requerimientos de la población geriátrica, con los antecedentes y diagnósticos que más frecuentemente presentan y de su tratamiento en particular ${ }^{4,5,6}$.

En la mayor parte de las ciudades de nuestro país, los establecimientos de salud se encuentran saturados, más aún en los servicios de urgencias y emergencias, debido probablemente al desigual crecimiento de la población que tiene acceso a estos y la cartera de servicios de salud que se brindan, problemática que al parecer no ha sido prevista ni afrontada a tiempo. Las poblaciones en las zonas urbanas crecen exponencialmente, los altos índices de violencia, delincuencia y la generalización del pandillaje juvenil se traducen en altas cifras de víctimas con graves lesiones, potenciándose con un caótico crecimiento vehicular y la aparición de modalidades de transporte como son los mototaxis?.

El hacinamiento en los servicios de emergencia traduce la problemática de los diversos sistemas de salud. Hoy en día, los principales problemas que aquejan a la población mayor de 60 años son los relacionados a patologías crónico-degenerativos, donde las enfermedades cardiovasculares están en el primer orden, lo que conduce a una mayor utilización de los servicios de salud, en particular las Emergencias, mayor costo de la atención y mayor capacidad resolutiva de los establecimientos de salud ${ }^{8,9}$.

Lo mencionado crea una necesidad de servicios de salud que deben ofrecer calidad, oportunidad y eficacia en la atención del paciente crítico de emergencia en concordancia con las nuevas concepciones de atención en salud.

Uno de los aspectos más importantes tanto a nivel hospitalario como del propio servicio de emergencia en razón a la percepción de calidad de atención de los pacientes es el primer contacto que realizan los mismos con el hospital. El constante incremento del número de atenciones en los servicios de urgencias o emergencias hospitalarios determina la necesidad de disponer de un sistema para clasificar pacientes que acuden a dichos servicios, en función de su gravedad probable, para atender de forma prioritaria a quien más lo amerita, este proceso es el Triage y el lugar donde se realiza el primer contacto de los usuarios de los servicios de emergencia con el personal de salud son las áreas de Triage las que pueden estar organizadas en mayor o menor medida en los servicios de emergencia.

Triage, significa clasificación de pacientes según su estado de salud. El triage es un proceso de valoración clínica inicial que clasifica a los pacientes antes de la evaluación diagnóstica y terapéutica medica, en base a su grado de urgencia, sin necesariamente tomar en cuenta el orden de llegada, determinando que los pacientes más urgentes sean evaluados primero mientras los restantes deben ser controlados y reevaluados hasta su evaluación médica completa ${ }^{10}$.

\section{Historia del triage}

El desarrollo del Triage nace de las experiencias de la guerra y medicina militar, en épocas antiguas los 
ejércitos realizaban escaso o ningún esfuerzo para atender a sus heridos por el nivel de sus conocimientos, las pocas atenciones brindadas eran poco o totalmente inefectivas ${ }^{11}$.

El cirujano militar francés barón Dominique-Jean Larrey, cirujano jefe de la Guardia Imperial de Napoleón es considerado por la mayoría de autores como el primero en concebir y poner en práctica un sistema oficial de triage en el campo de batalla a través de la evaluación y clasificación rápida de los soldados heridos $^{11,12}$.

Según señala Iserson ${ }^{12}$, priorizó el tratamiento y la evacuación de heridos que exigían cuidados médicos urgentes en lugar de la espera de horas y hasta días o que la batalla termine. De esa manera, Larrey realizó cientos de amputaciones en el campo de batalla mientras que la batalla se desarrollaba, y también diseñó carros ligeros, que denominó "ambulancias voladoras" para transportar rápidamente el herido. Decía "los que están peligrosamente heridos deben recibir la primera atención, sin importar rango o distinción, los que se lesionan en un grado menor pueden esperar hasta que sus hermanos de armas, que están muy mutilados, hayan sido operados y vestidos, de lo contrario éstos no sobrevivirán durante muchas horas, rara vez, hasta el día siguiente" lo que quedó plasmado en sus memorias sobre la campaña de Rusia ${ }^{18-12}$.

El cirujano naval británico John Wilson sostenía que los cirujanos deberían centrarse en aquellos pacientes que necesitaban tratamiento inmediato con mayor probabilidad de éxito; los pacientes con heridas menos graves y aquellos cuyas heridas eran probablemente fatales el tratamiento se prorrogaba.

Durante la Guerra Civil de los Estados Unidos (EEUU), con el establecimiento formal de un servicio de ambulancia de campo militar y estaciones de ayuda directas a través de los esfuerzos de dos cirujanos del Ejército de la Unión, Charles Tripler y Jonathan Letterman se consolidó lo señalado por Larrey ${ }^{11}$.

Sin embargo, en los primeros días de la Guerra civil en EEUU no existía un método uniforme de clasificación de los heridos en combate determinando alta mortalidad lo que devino en la implementación de procedimientos de selección en la primera línea y servicios de ambulancia disminuyendo la mortalidad.

Con el advenimiento de la primera guerra mundial se desarrolló un número de armas de alta letalidad con gran número de víctimas no visto antes, que necesitando tratamiento debían entrar a un sistema de triage. Como refiere Iserson ${ }^{12}$, Keen en su libro "Tratamiento de las heridas de guerra" menciona que "un hospital con 300 o 400 camas derrepente puede ser abrumado por 1000 o más casos. A menudo, por lo tanto, es materialmente imposible dar un tratamiento rápido y completo a todos. Un solo caso, incluso si se requiere con urgencia de atención y si va a absorber mucho tiempo, podría tener que esperar, ya que en ese mismo tiempo una docena de otros, casi igual de exigentes, pero que requieren menos tiempo, podrían ser atendidos. El mayor bien para el mayor número debe ser la regla".

Este enfoque reconoce explícitamente que, cuando los recursos son limitados, algunos pacientes que podrían salvarse pueden esperar mientras se salva a mayor número. Otros planificadores de triage en la primera Guerra ofrecen un enfoque muy diferente al triage del campo de batalla, que plantea retrasar el tratamiento de los menos gravemente heridos, ya que sugirieron dar prioridad a este grupo, pues pueden ser tratados con rapidez y volver al combate.

En la Segunda Guerra Mundial se introdujeron nuevas armas, incluyendo tanques mejorados y apoyo aéreo, y nuevos tratamientos, incluyendo plasma y la penicilina. Médicos militares desarrollaron protocolos nuevos y más detallados para la evaluación y triage de pacientes.

Del mismo modo, los médicos militares alemanes, durante la campaña rusa de 1941, utilizaron el principio de maximizar la fuerza de combate por el tratamiento de aquellos que podrían más rápidamente volver a la acción con el menor gasto de tiempo y recursos.

Otro ejemplo de este enfoque de triage militar se puede encontrar en un manual de 1958 de la OTAN (Organización del Tratado del Atlántico Norte) que describe tres categorías de triaje: 1. Aquellos que están heridos leves y pueden volver al servicio, 2. Aquellos que están más gravemente heridos y con necesidad de reanimación inmediata o cirugía, y el "irremediablemente herido" o muertos al arribo.

La evacuación rápida de los heridos empezó con el transporte aeromédico básico (sin atención médica en el aire) en la Guerra de Corea y avanzó a transporte sofisticado en helicóptero multicausal con tratamiento aéreo en Vietnam. El tiempo medio entre la lesión y el tratamiento definitivo se redujo de 12 a 18 horas en la Segunda Guerra Mundial, de 2 a 4 horas en Corea, y menos de 2 horas en Vietnam. En los 2 conflictos de Irak, hospitales de campaña móviles, idealmente dentro de 10 millas del campo de batalla, se mantuvo tiempos de evacuación relativamente cortos. 
El uso de armas nucleares en la Segunda Guerra Mundial y la continua amenaza de armas nucleares, químicas y biológicas de destrucción en masa plantean desafíos especiales para la clasificación y la planificación de triage. En un ataque limitado con las armas de destrucción masiva, la planificación de triage para las grandes catástrofes puede ayudar a los proveedores a distribuir los recursos limitados entre los supervivientes heridos. Después de que el uso generalizado de este tipo de armas o un desastre natural de gran magnitud, el número de víctimas, la destrucción de los recursos disponibles y del orden social puede ser tan grande que el cuidado médico efectivo, incluyendo triage, se hace imposible.

Se menciona a menudo que los sistemas militares de triage se han adaptado para triage en contextos civiles, incluidos los desastres. Con base en una revisión exhaustiva de los desastres de Estados Unidos, Auf der Heide informó de que, a pesar de la existencia de sistemas de triage, la mayoría de las víctimas de desastres fuera del hospital, se encuentran y se transportan directamente a los hospitales por los transeúntes ${ }^{13}$.

La primera descripción del uso civil del triage en los Departamentos de Emergencia (DE) por primera vez fue en Yale-New Haven Hospital en 1963, con un enfoque hacia la población civil y fue publicada por Weinerman en $1964^{14}$. Su clasificación incluía tres categorías:

a) Emergente (condiciones que requieren atención médica inmediata, ya que ponen en riesgo la vida).

b) Urgente (condiciones que requieren atención médica dentro de un periodo de pocas horas por considerarse un padecimiento agudo, pero no necesariamente grave).

c) No urgente (condición que no amerita el uso de los recursos del Departamento de Urgencias).

Basado en las necesidades de los pacientes y el personal de salud que recibe en primer lugar a los pacientes que acuden a Emergencia, dicha clasificación fue modificándose con la experiencia y los diversos trabajos de investigación desarrollados, por lo que en la actualidad se plantea un triage con escala de cinco niveles en base a la priorización en razón a la gravedad presentada ${ }^{15}$.

- Nivel I: prioridad absoluta con atención inmediata y sin demora.

- Nivel II: situaciones muy urgentes de riesgo vital, inestabilidad o dolor muy intenso. Demora de asistencia médica de hasta 10 minutos.
- Nivel III: urgente pero estable hemodinámicamente, con potencial riesgo vital que probablemente exige pruebas diagnósticas y/o terapéuticas. Demora de atención de 60 minutos.

- Nivel IV: urgencia menor, potencialmente sin riesgo vital para el paciente. Demora máxima de 120 minutos.

- Nivel V: No urgencia. Poca complejidad de la patología o cuestiones administrativas, citas, etc. Demora de hasta 240 minutos.

Con base en el sistema de cinco prioridades antes mencionado, actualmente existen cinco modelos de triage hospitalario de uso universal ${ }^{16}$, existiendo diversos intentos locales para implementar sus propios modelos $17,18,19$.

\section{Modelos de triage hospitalario}

a) Escala de triage Australiana, NTS de 1993 y ATS 2000

b) Triage del Departamento de Urgencias de Canadá CTAS de 1995

c) Sistema de triage de Manchester MTS de 1996,

d) Índice de Severidad de urgencia ESI de 1999 y

e) Sistema de triage Andorrano o Sistema español de Triage MAT-SET 2003

El modelo de clasificación debe ser estandarizado, normalizado y tener un alto nivel de concordancia interobservador, válido al clasificar al paciente según su nivel real de urgencia. El objetivo primario de la clasificación será optimizar el lapso de tiempo desde que el paciente llega a emergencia y es valorado por el médico que atenderá al paciente.

La prioridad que se asigne dependerá del motivo de consulta, de la valoración clínica, de los signos objetivos y subjetivos del paciente, este proceso es continuo hasta iniciar la atención médica en el tópico respectivo.

Durante el proceso de triage se pone de manifiesto el principio de la beneficiencia, es decir conseguir lo mejor para el paciente con uso racional de los recursos existentes, así como el principio de justicia con asignación de dichos recursos a los que tengan mayores posibilidades de sobrevida.

Los antecedentes del sistema de cinco prioridades se originan en Australia. Como señala FitzGerald ${ }^{20}$, en Australia el desarrollo de un sistema de clasificación se basó en las observaciones de las enfermeras en la clasificación de los pacientes. Esta observación identificó varias acciones consistentes y distintas después de la evaluación las que fueron determinadas 
por la urgencia del paciente e incluyeron:

1.Solicitar atención médica de inmediato y comenzar la reanimación.

2.Asignar al paciente al siguiente médico disponible.

3. Colocar la historia de emergencia del paciente al frente de la lista de espera.

4. Colocar la historia de emergencia del paciente en orden en la lista de espera.

5. Animar al paciente a buscar ayuda en otro lugar o en otro momento.

La decisión central que subyace a estas observaciones fue la "urgencia" del paciente y la evaluación de la enfermera del momento en que debe realizarse la evaluación médica. Esto condujo al desarrollo del The Ipswich Triage Scale (ITS), que se basó en gran medida en una escala categórica de 5 niveles en funcionamiento en el Hospital Box Hill en Victoria. Sin embargo, el ITS incluyó un descriptor de urgencia funcional basado en la determinación general de la enfermera de la urgencia del paciente.

En el ITS el paciente debe ser visto bajo circunstancias razonables por un médico en el plazo de: 1 . Segundos, 2. Minutos, 3. Una hora, 4. Horas y 5. Días. La categoría de "días" no implicaba que los pacientes debían esperar días, sino que podían hacerlo sin efectos clínicos adversos.

Después de esto, se realizó un análisis detallado del uso de la escala durante 12 meses en el Hospital Ipswich. Estos estudios mostraron un nivel relativamente alto de concordancia en las evaluaciones de clasificación entre enfermeras, una relación directa entre la evaluación de clasificación y una variedad de otras medidas de gravedad (p. Ej., Score de trauma, Score de gravedad de lesiones y Score de gravedad de asma) y una asociación directa con resultados tales como la mortalidad, el tiempo en el hospital, el tiempo en la $\mathrm{UCl}$ y la utilización de recursos.

Otros estudios realizados por Jelinek ${ }^{21}$ confirmaron la repetibilidad y la validez del sistema. Este trabajo constituyó la base de la adopción del ITS en 1994 por el Australasian College for Emergency Medicine (ACEM) como la Escala Nacional de Triage (NTS).

McMahon $^{22}$ señala que el ATS requiere que el personal de salud capacitado evalúe a todos los pacientes a su llegada, determinando la categoría de clasificación al completar la oración: "Este paciente debe esperar para recibir una evaluación médica y un tratamiento de no más de minutos". Cada categoría de clasificación tiene un tiempo máximo recomendado para el tratamiento. La evaluación toma de dos a cinco minutos; Las enfermeras obtienen signos vitales y otros datos solo cuando es necesario. Un amplio desarrollo de esta escala se puede obtener de https://acem. org.au/Content-Sources/Advancing-EmergencyMedicine/Better-Outcomes-for-Patients/Triage.

Posteriormente, el NTS se convirtió en el Australasian Triage Scale - ATS en 2000 y ha sido ampliamente validado por la experiencia de investigación y operativa., el ATS también es una de las bases del Canadian Triage and Acuity Scale CTAS en Canadá y el Manchester Triage System MTS del Reino Unido.

McMahon $^{22}$, señala que la escala canadiense de clasificación y agudeza (CTAS) es similar a la ATS pero tiene pequeñas diferencias en el tiempo para la evaluación del médico. También incluye escalas de dolor y una escala de agudeza separada para pacientes pediátricos, y permite la determinación de la gravedad de las afecciones (como el asma) en más de una categoría, y tiene un "resumen de vista rápida" de las categorías de clasificación desglosadas por sistema corporal. Además, describe los marcos de tiempo para la reevaluación de los pacientes en el área de espera y permite cambiar la categoría de clasificación cuando el paciente ha estado esperando durante mucho tiempo (por ejemplo, un paciente clasificado como " 5 " puede cambiar a un "4" después de esperar dos horas). También tiene un indicador de calidad que mide si el departamento cumple con sus propios estándares de tiempo de respuesta. Se puede obtener ampliamente desarrollada en https://caep.ca/ resources/ctas/implementation-guidelines/

El Sistema de Triage de Manchester, desarrollado por el Grupo de Trabajo de Triage de Manchester, Inglaterra, dirige al personal profesional de salud de triage para asignar niveles mediante la identificación de signos y síntomas y la evaluación de seis factores: amenaza para la vida, dolor, hemorragia, nivel de conciencia, temperatura y agudeza. (Evita específicamente el diagnóstico durante el triaje). Se han desarrollado cincuenta y dos algoritmos para su uso durante este proceso. Su desarrollo se puede ver en https:// www.triagenet.net/classroom/ o en http://www. triagemanchester.com/web/presentacion_es_66.php

El Índice de gravedad de emergencia (ESI), desarrollado por un equipo de médicos de emergencia y enfermeras dirigidos por Wuerz, ya fallecido, en Brigham and Women's Hospital en Massachusetts y por Eitel en York Hospital en Pensilvania. EI ESI se basa en un modelo conceptual que no solo plantea la pregunta 
"¿Cuándo debería verse a este paciente? " pero también “ ¿Qué necesita este paciente? " por lo tanto, el algoritmo primero incorpora la agudeza y luego el consumo de recursos esperado para determinar la prioridad del tratamiento. Los componentes incluyen la estabilidad de la vía aérea, la función cardíaca, el nivel de conciencia, la intensidad del dolor, el número de intervenciones requeridas y el nivel de riesgo. Se ha probado su alto grado de confiabilidad entre los evaluadores en la categoría de clasificación. También se observó una fuerte correlación entre la categoría de clasificación y la probabilidad de hospitalización. La versión 4 con ejemplos se puede obtener de https:// www.ahrq.gov/professionals/systems/hospital/esi/ index.html

El Sistema Español de Triaje - Modelo Andorrano de Triaje (SET-MAT), surge en el año 2000, con el CTAS como referente, pasando de una escala basada en síntomas y diagnósticos centinela a una escala basada en categorías sintomáticas. Es un conjunto de síntomas o síndromes que el profesional reconoce a partir del motivo de consulta y permite clasificarlo en una categoría sintomática ${ }^{10}$.

En el año 2001, se desarrolló el programa informático de ayuda al Triaje y la Sociedad Española de Medicina de Urgencias y Emergencias (SEMES) lo adopta como modelo de triaje para el estado español en el 2003, con el nombre de Sistema Español de Triaje (SET). EI SET posee 32 categorías sintomáticas y 14 subcategorías que agrupan 578 motivos de consulta diferentes.

EI SET-MAT utiliza una escala de prioridad de 5 niveles y posee una versión específica para pediatría con unas categorías sintomática más concreta según la población empleada. Está versión ha sido validada, aunque no muestra una correlación tan estrecha entre consumo de recursos de transporte sanitario o índice de ingresos según el nivel de urgencia, como en la población adulta.

\section{Principios del triage}

Según la OPS en su Manual para la implementación de un sistema de triage para los cuartos de Urgencias ${ }^{23}$, los principios fundamentales que caracterizan a un sistema de triage estructurado y moderno son:

- Sistema de triage de 5 niveles de priorización, normalizado y dotado de un programa informático de gestión del triage, que permita el registro de la clasificación, el control de todos los pacientes dentro y fuera del servicio y el control de tiempos de actuación ("Timelines").
- Modelo integrador de los aspectos más relevantes de los modelos actuales de triage de 5 categorías, a los que ha de aportar aspectos de revisión y adaptación al entorno sanitario donde se aplique.

- Modelo de calidad, con objetivos operativos, propuestos como indicadores de calidad del triage, fiable, válido, útil, relevante y aplicable.

- Sistema de triage médico y de enfermería no excluyente, integrado en una dinámica de servicio donde se prioriza la urgencia del paciente, sobre cualquier otro planteamiento estructural o profesional, dentro de un modelo de especialización de urgencias.

- Modelo dotado de un programa informático de ayuda a la decisión clínica en el triage, evaluado y validado, con ayuda continua y con registro anamnésico del triage.

- Sistema integrado en un modelo de mejora continua de la calidad, con monitorización de indicadores de calidad del triage, que defina un estándar de motivos de consulta a urgencias y que permita entre otros, evaluar la casuística del servicio.

- Poder integrar en un modelo global de historia clínica electrónica, integradora de la actividad médica y de enfermería, estandarizada y acorde con estándares de calidad, que permita un total control de la gestión clínica y administrativa del cuarto de urgencias.

- Proponer adaptaciones estructurales y de personal en el cuarto de urgencias, acordes con las necesidades de calidad del sistema de triage y formación específica para el personal de triage.

- Sistema de triage estructurado holístico, de aplicación tanto en el terreno de la urgencia hospitalaria como extrahospitalaria, aplicable tanto a niños como a adultos, y con independencia del tipo de hospital, dispositivo o centro de asistencia.

\section{Objetivos del triage}

Los objetivos del sistema de triage en Emergencia ${ }^{15}$ apuntan hacia la identificación oportuna, rápida de los pacientes en situación de riesgo vital, intenta disminuir la congestión de pacientes asegurando el otorgamiento de la prioridad adecuada destinándolo al área más adecuada para tratar el caso. Como ya se ha señalado no es la intención establecer diagnósticos sino otorgar la prioridad de atención.

Un aspecto importante es tener como objetivo la información a los pacientes y sus familiares sobre el estado general del paciente y de los tiempos probables de espera. 


\section{Ventajas del triage}

El triage proporciona al paciente información sobre su estado de salud y tiempo aproximado de espera priorizando su atención de acuerdo a su gravedad clínica. Asimismo, orienta los flujos de paciente en función a su gravedad permitiendo la reordenación de los recursos de emergencia en función de la demanda.

\section{Funciones del triage}

Determinar la situación de riesgo vital del paciente, mediante sistema de prioridades de atención, determinando el área más adecuada para tratar al paciente informando a los pacientes y familiares de las primeras acciones a realizar según su prioridad de atención y el tiempo de espera probable.

\section{Indicadores de calidad del triage}

La clasificación de un triage estructurado permite tener índices de calidad. Algunos de ellos son el tiempo de espera para ser atendido en el triage el que debe ser, en general, menor de 10 minutos. También es índice medible la proporción de pacientes que dejan el hospital sin ser atendidos por un médico y que, en general, debe ser menor del $2 \%$.

Se ha planteado ${ }^{10-24}$ indicadores de calidad para el triage hospitalario los cuales serían:

1. El índice de pacientes perdidos sin ser visitados por el médico que sería un Índice de Calidad de satisfacción, riesgo y adecuación. El estándar establecido se sitúa en $2 \%$ del total de pacientes que acuden a urgencias, lo subdividen en:

a. Índice de pacientes perdidos sin ser clasificados que es el porcentaje de pacientes que deciden dejar el servicio de urgencias después de ser registrados administrativamente y antes de ser clasificados, sobre el total de pacientes registrados.

b. Índice de pacientes clasificados y perdidos sin ser visitados por el médico que es el porcentaje de pacientes clasificados que deciden dejar el servicio de urgencias antes de ser visitados por el médico, sobre el total de pacientes clasificados.

2. El tiempo desde la llegada del paciente al servicio de urgencias hasta el momento que se inicia la clasificación. Se recomienda que este tiempo sea de 10 minutos, este índice no tiene un estándar establecido, es difícil de calcular salvo haya un sistema electrónico y automático de registro de llegada.

3. El tiempo de duración de la clasificación (tiempo de duración del triaje), se recomienda que sea 5 minutos.
4. El tiempo de espera para ser visitado, se establece que al menos un $90 \%$ de los pacientes tienen que ser visitados por el equipo médico en 2 horas desde su clasificación y el 100\% en 4 horas.

Los diversos tipos de modelos de triaje, deben tener fiabilidad y reproducibilidad altas. La fiabilidad es un término estadístico que evalúa el grado de uniformidad interevaluador e intraevaluador. Ya sea que dos personas distintas o la misma persona realicen el triage obtendrían el mismo resultado ${ }^{25}$.

En el momento actual un triage correcto se realiza a través de la informatización del mismo pues a pesar de la fiabilidad y validez científica de las escalas de triage de 5 niveles, la experiencia de su uso ha mostrado problemas traducidos en trabajos de investigación como fiabilidad de los resultados en la práctica clínica (problemas de concordancia interprofesional e ínterhospitalaria cuando se aplica sobre pacientes en el triage), atribuidos a diferentes causas: 1.- heterogeneidad en la formación de los profesionales; 2 .- tendencia al subtriage en cuartos de urgencias sobresaturados; 3.- tendencia al subtriage en situaciones agudas comunes; 4.- tendencia al sobretriage para justificar largos tiempos de demora; 5.- tendencia al sobretriage en servicios remunerados por casuística. Todo lo cual disminuiría con una informatización del triage que se constituiría en un soporte fundamental para el personal del triage y asimismo permite una auditoria más fiable ${ }^{23}$.

La implementación en los servicios de emergencia del área de triage ha determinado en parte, satisfacción por parte del personal de urgencias, dado que la patología que afronta ha sido seleccionada y calificada antes de su atención, disminuyendo aquella que es la que no debería atender y pero que si debe recibir atención médica en otros niveles de asistencia.

Se produce una educación sanitaria de la comunidad, haciendo que el paciente con patología banal, que no requiere estudios ni pruebas complementarias hospitalarias y que piensa que por acudir a los servicios de urgencias hospitalarias le van a realizar estos exámenes auxiliares, se encuentre con un necesario triage que discrimina esa situación. Asimismo, se ha conseguido una mejora en la calidad asistencial dado que se atiende de manera adecuada y en el tiempo necesario aquellas patologías que hacen necesario una prontitud en su asistencia, aumenta la satisfacción de los usuarios externos e internos y racionaliza el consumo de recursos colaborando en la mejora de la calidad global del servicio y aportando un orden 
justo en la asistencia, basado en la gravedad, de los pacientes, emergencia /urgencia ${ }^{26,27}$.

Dentro de la organización del triage, se ha estudiado qué profesional podría ejecutarlo aparte del Médico. Imperato, en un estudio de intervención de 6 meses antes y después de implementar la presencia del médico afirma que mediante una encuesta dicha presencia tuvo una significativa mejora en la satisfacción del paciente $26-28,29,30$.

Rainer, en un estudio observacional prospectivo de pacientes consecutivos que asistieron a la estación de triage de un servicio de urgencias entre 9am y $5 \mathrm{pm}$, de lunes a viernes, evaluó el impacto de un especialista en el triage en tiempo de servicio. Cuando se compararon las diferentes categorías de triage, reducción significativa sólo se observó en pacientes semiurgentes durante el período de estudio. Sin embargo, los pacientes semiurgentes constituyen el $68 \%$ de todas las asistencias en el grupo de estudio y $74 \%$ en el grupo control, concluyendo que la presencia de un especialista de medicina de Emergencia puede acortar el tiempo de servicio de los pacientes de categoría 4 y que el triage de pacientes puede realizarse mejor por un equipo de triage con la presencia de un especialista en medicina de Emergencia y enfermeras en lugar de solo una enfermera de triage ${ }^{31}$.

Por otro lado, las decisiones tomadas pueden tener un margen de error que es necesario monitorear, como lo publica Grossmann al informar sobre un"subtriage" que se produjo en 117 casos de un total de 519 pacientes de 65 o más años, siendo las razones principales las situaciones de alto riesgo y la falta de interpretar adecuadamente los signos vitales concluyendo que en su estudio, los pacientes mayores corrían riesgo de "subtriage". Fue realizado con el índice de gravedad de emergencia o Emergency Severety Index al que sin embargo encontraron confiable y válido para el triage de pacientes mayores ${ }^{32}$.

Una revisión de búsquedas en las bases de datos de estudios comparativos publicado de 1994 a 2014 que examinan el papel del médico experimentado en triage, demuestra que el triage realizado por médicos experimentados puede ser una medida efectiva para mejorar el rendimiento del departamento de emergencia ${ }^{33}$.

La preocupación por la sensibilidad del triage también se vio revelado en otro trabajo de Mendoza y Elguero quienes determinaron la sensibilidad del triage clínico en un servicio de urgencias adultos realizando un estudio prospectivo transversal y clínico, incluyendo pacientes mayores de 18 años que acudieron a solicitar consulta al servicio de urgencias y fueron valorados por el médico del servicio de triage. La muestra fue de 380 y los pacientes que ingresaron fueron aleatorizados. Se utilizó el sistema de 3 colores, y posterior a su atención médica se volvió a asignar un color dependiendo de su diagnóstico final, comparándose con el inicial para determinar la sensibilidad diagnóstica. De 380 pacientes clasificados inicialmente en el área de triage, obtuvieron una sensibilidad diagnóstica global de 60.2 concluyendo que la sensibilidad del triage clínico en el Servicio de Urgencias Adultos de su hospital fue de 60.2, y que la sensibilidad para los pacientes con urgencia real es mejor que la sensibilidad para los pacientes con urgencia media y sentida ${ }^{34}$.

Por otro lado, Beveridge reporta que hubo alta tasa de acuerdo interobservador usando la Canadian Triage and Acuity Scale y fue asimismo entendida e interpretada de manera similar por enfermeras y médicos ${ }^{25}$.

La sobredemanda en emergencia y el cambio demográfico que determina la asistencia de mayores de la tercera edad a la emergencia, es preocupación de varias realidades como lo traduce Baumann en su trabajo sobre Triage de pacientes geriátricos en un departamento de emergencia y la validación y sobrevida con el índice de severidad en emergencia donde evaluaron la validez de dicho índice y su asociación con la sobrevida. Cuando se utiliza en el triage para los pacientes mayores de 65 años, el algoritmo ESI demuestra validez, la hospitalización, duración de la estancia, utilización de recursos y supervivencia fueron asociadas con la categorización de la ESI en esta cohorte, y se usó en este grupo de pacientes pues existe una gran demanda de la Emergencia por este grupo etáreo en diversas latitudes, incluyendo nuestro país ${ }^{35}$.

Sobre la investigación en Triage, las escalas de triage de cinco niveles son superiores a los sistemas de tres niveles con respecto a la validez y fiabilidad por lo que el mayor número de publicaciones se refieren a los instrumentos de cinco niveles CTAS y ESI, los cuales han sido objeto de estudios multicéntricos y análisis realizados en Europa. El ATS es también bien documentado, pero son escasos los datos publicados en el MTS. La capacitación constante permite a los profesionales que realizan triage un nivel óptimo en sus decisiones incrementando sus competencias y la seguridad percibida al realizarlo ${ }^{36,37}$.

Uno de los aspectos observados en el área de triage es el insuficiente o excesivo triage. Al respecto, la OPS 
a través de la publicación "desarrollo de sistemas de servicios de emergencias médicas: experiencia de los Estados Unidos de América para países en desarrollo"38 menciona lo siguiente: Se denomina triage insuficiente a la situación en la cual la enfermedad o la lesión de un paciente es catalogada como menor que la que en realidad tiene, esto puede dar lugar a alguno o todos de las siguientes situaciones: mayor probabilidad de muerte, mayor probabilidad de discapacidad permanente, aumento de los costos por pérdida de productividad $\mathrm{y}$, en último término, mayores costos de la atención médica como resultado del retraso del tratamiento de la enfermedad o lesión, o sus complicaciones resultantes. Por el contrario, se llama triage excesivo a la situación en la cual la enfermedad o la lesión de un paciente es catalogada como más grave que la que en realidad tiene, las consecuencias son, sin embargo, paradójicamente similares a las anteriores, aumento de los costos médicos secundarios al uso innecesario de recursos médicos (personal, pruebas y equipo).

Además, el hecho es que mientras los pacientes erróneamente catalogados utilizan los recursos de Servicio de Emergencias Médicas o del Departamento de Emergencia, alguien con un cuadro más grave no puede hacerlo. Esto hará que el paciente quizás "desconocido" en el sistema sufra el efecto de las consecuencias del "triage insuficiente" ya mencionado, lo mencionado sin duda puede ser de naturaleza cíclica, causando un efecto de propagación en el departamento de emergencia.

También refiere que "en la mayoría de los sistemas, especialmente en lo que concierne a la población de pacientes con traumatismos, se acepta universalmente que un cierto porcentaje de triage excesivo contra un triaje insuficiente es aceptable, puesto que el error apunta a la cautela. En los traumatismos, se acepta en general que una tasa de triaje excesivo de $30 \%$ a $50 \%$ es aceptable (Colegio Estadounidense de Cirujanos, 1999).

Es aconsejable, sobre una base moral y ética, lograr un equilibrio entre proporcionar atención de alta calidad a todos y hacerlo con costos razonables, el objetivo es lograrlo de modo tal de NO infringir las normas de calidad respecto de los pacientes que están verdaderamente necesitados de atención inmediata y urgente. Este es el desafío real que enfrentan los sistemas de triage de los SEM y del DE en el nuevo milenio"38.

Si se ha decidido implementar un sistema de triage, debe seleccionarse un instrumento cuya validez y fiabilidad ha sido demostrada, idealmente en la lengua del país de que se trate, además, que permita la correcta identificación de los pacientes que requieren atención médica urgente; estos instrumentos facultan estimación y planificación de recursos ${ }^{39}$.

\section{Triage en los servicios de emergencia de Lima}

En el Perú en general y en Lima en particular, los cambios no se han dado de manera progresiva $y$ continua, sino más bien se han sobrepuesto varias etapas como producto del cambio epidemiológico y demográfico ocurrido en los últimos 30 años y donde la violencia del terrorismo y de pandillas así como los rápidos cambios en el parque automotor y las enfermedades crónico-degenerativas han determinado una sobredemanda de los servicios de emergencia. La respuesta del sistema de salud no ha sido oportuna.

La mayoría de los servicios de emergencia para enfrentar esta sobredemanda han potenciado áreas como el triage, pero de manera desordenada y sin fundamentos normativos generales y específicos, lo mencionado se ve reflejado preliminarmente en un estudio sobre triage en los hospitales de Lima; se evaluaron 20 servicios de emergencia hospitalario, $65 \%$ tenía por categorización el nivel III-1, 25\% nivel II-2, 5\% nivel IIE y $5 \%$ el nivel II-1. El tiempo de inicio del triage en sus servicios de emergencia es en promedio de 8.61 años, funcionan en promedio las 24 horas en $60 \%$ de establecimientos de salud y de lunes a domingo en $70 \%$ de ellos.

La prioridad usadas son de 4 en un $61.11 \%, 5$ prioridades en $33.3 \%$, y 3 prioridades en $5.56 \%$. El triage en $70 \%$ está a cargo del profesional médico y el 30\% de enfermería. Hay 15\% (3 establecimientos) realizado por técnicos de enfermería de manera parcial.

La programación médica es solo total sólo en $30 \%$ de los establecimientos y parcial en 30\%. 8 establecimientos no informaron acerca del tipo de programación. 15\% del total de 20 establecimientos informan que el médico que realiza triage tiene especialidad, niegan que tengan especialidad 11 establecimientos y 6 no informan. Enfermería presenta un $83.33 \%$ que tienen especialidad. En $90 \%$ se informa a familiares o pacientes sobre la situación de salud y $65 \%$ es informado sobre los tiempos de espera. 20\% maneja indicadores de calidad y $10 \%$ realiza retriaje. $50 \%$ de establecimientos de salud usan escalas de triaje y $85 \%$ del personal de los establecimientos que las usan tienen conocimiento de las mismas. $85 \%$ de establecimientos de salud informa que tienen normativa interna sobre triage y $75 \%$ del personal conocería las mismas. 90\% de establecimientos informan que no realizan investigación en el área de triage $^{40}$. 
Todo ello presenta un panorama de inadecuada implementación del área de triage en servicios de Emergencia de establecimientos de salud de Lima por lo que en un entorno de mejora de la calidad asistencial y en el objetivo de mejorar la atención del paciente crítico se debería establecer normas del ente rector en salud a fin de uniformizar conceptos modernos sobre triage e implementar un área tan importante y en muchos casos decisiva en la primoatención del paciente de emergencia.

\section{CONCLUSIÓN}

El triage determina la situación de riesgo vital del paciente, proporciona al paciente información sobre su estado de salud y del tiempo aproximado de espera de acuerdo a su gravedad clínica. El triage debe ser realizado un sistema de triage estructurado y moderno.

\section{REFERENCIAS BIBLIOGRÁFICAS}

1.- Alagappan K, C. Holliman J. History of the Development of Internationa
Contribuciones de autoría: Los autores participaron en la generación, recolección de información, redacción y aprobación final del artículo original.

Financiamiento: Autofinanciado.

Conflicto de interés: Los autores declaran no tener conflicto de interés en la publicación de este artículo.

Recibido: 01 de noviembre del 2018

Aprobado: 02 de diciembre del 2018

Correspondencia: Rolando Vasquez Alva.

Dirección: Cápac Yupanqui N²698-104, Lince. Lima, Perú

Celular: 999952704

Correo: rolandova@hotmail.com Emergency Me dicine, Emerg Med Clin N Am 23 (2005) 1-10

2.-Luna Rocha J. Historia de la medicina de urgencia en Mexico Archivo de medicina de Urgencia de Mexico Vol 1 Num 1 Mayo-agosto 2009 pp 4-11

3.-Instituto Nacional de Estadistica e Informatica- https://www.inei.gob. pe/media/MenuRecursivo/publicaciones_digitales/Est/Lib1530/libro.pdf

4.-Torné $E$, et al. Análisis de la demanda en los servicios de urgencias de Barcelona. Aten Primaria 2003:32(7):423-429

5.-McConnel CE,Wilsnon RW. The demand for prehospital emergency services in an aging society. Soc Sci Med 1998;46:1027-1031

6.- Vásquez-Alva R, Amado-Tíneo J, Ramírez-Calderón F, VelásquezVelásquez R, Huari-Pastrana R. Sobredemanda de atención médica en el servicio de emergencia de adultos de un hospital terciario, Lima, Perú. An Fac Med. 2016;77(4):379- 85. doi 10.15381/anales.v77i4.12654

7.- Morales-Soto N. Algunas consideraciones para la organización de servicios de salud para emergencias y desastres. An Fac med. 2001;62(1):44-55.

8.-Hoot N, Aronsky D. Systematic Review of Emergency Department Crowding: Causes, Effects, and Solutions Ann Emerg Med. 2008;52:126-136

9.-Pereda J, Díaz I, Pereda R, Sosa A. "Filtro sanitario" en las urgencias médicas. Un problema a reajustar Rev Cubana Med 2001;40(3):181-188

10.-Gómez Jiménez J. Clasificación de pacientes en los servicios de urgencias y emergencias: Hacia un modelo de triaje estructurado de urgencias y emergencias. Emergencias 2003;15:165-174

11.-Mitchell GW. A Brief History of Triage. Disaster Medicine and Public Health Preparedness. Cambridge University Press; 2008;2(S1):S4-S7. doi:10.1097/DMP.0b013e3181844d43

12.-Iserson K. Moskop J. Triage in Medicine, Part I: Concept, History, and Types Annals of Emergency Medicine Volume 49 N 3 March 2007

13.- Auf der Heide E. The importance of evidence-based disaster planning. Ann Emerg Med. 2006; 47:34-46.

14.- Weinerman ER, Ratner RS, Robbins A, et al. Yale studies in ambulatory care V: determinants of use of hospital emergency services. Am J Public Health. 1966;56:1037-1056
15.-López RJ, Montiel EMD, Licona QR. Triage en el servicio de urgencias. Med Int Méx 2006; 22: 310-308.

16.- Cubero-Alpízar,C. (2014). Los sistemas de triage: respuesta a la saturación en las salas de urgencias. Rev. Enfermería Actual en Costa Rica, 27, 1-12. 2015 DOI: http://dx.doi.org/10.15517/revenf.v0i27.16145

17.- Pérez N, Nissen L, Nielsen RF, Petersen P, Biering K. The predictive validity of RETTS-HEV as an acuity triage tool in the emergency department of a danish Regional Hospital. Eur J Emerg Med 2016;23:33-7. 18.- Koyama T, Kashima T, Yamamoto M, Ouchi K, Kotoku T, Mizuno Y, A study of the effect of introduction of JTAS in the emergency room Acute Medicine \& Surgery Vol 4, 32017 262-270

19.- Taboulet, P., Moreira, V., Haas, L., et al., 2009. Triage with the French Emergency Nurses Classification in Hospital scale: reliability and validity. Eur. J. Emerg. Med. 16 (2) 61-67.

20.-Fitzgerald G, Jelinek G, Scott D, Gerdtz M: Emergency department triage revisited. Emerg Med J. 2010, 27: 86-92. doi:10.1136/ emj.2009.077081

21.-Jelinek GA. Casemix classification of patients attending hospital emergency departments in Perth, Western Australia. Development and evaluation o fan urgency-based casemix information system for emergency departments. Doctor of Medicine Thesis. University Western Australia 1995.

22.- McMahon MM. ED triage-is a five-level triage system best? Am J Nurs 2003;103:61-3.

23.- Organización Panamericana de la Salud/Organización Mundial de la Salud. Manual para la implementación de un sistema de triaje para los cuartos de urgencias. Washington D.C.:OPS/OMS2010 http://new. paho.org/hq/dmdocuments/2011/HSS_IS_Manual_Sistema_Tiraje_ CuartosUrgencias2011.pdf

24.- Hodge, A., Hugman, A., Varndekk, W., Howes, K. A review of the quality assurances processes for the Australasian Triage Scale (ATS) and implication for future practice. Australasian Emergency Nursing Journal. 2013. 16,21-29

25.- Beveridge R, Ducharme J, Janes L, Beaulieu S, Walter S. Reliability of the Canadian emergency department triage and acuity scale: interrater agreement. Ann Emerg Med 1999; 34:155-159. 
26.- Imperato J, Morris S, Sanchez D, et al. Improving patient satisfaction by adding a

physician in triage. J Hosp Adm 2014;3:7-13.

27.- Alvarez A. Gorostidi P. Rodríguez M. Estudio del triage y tiempos de espera en un Servicio de urgencias hospitalario. Emergencias Vol. 10, Núm. 2, Marzo-Abril 1998

28.- Janssen MA, van Achterberg T, Adriaansen MJ, Kampshoff CS, Schalk DM, Mintjes-de Groot J. Factors influencing the implementation of the guideline Triage in emergency departments: a qualitative study. J Clin Nurs. 2012;21:437-47

29.-Imperato J, Morris DS, Sanchez L, Setnik G Physician in Triage Improves Patient Satisfaction Annals of Emergency Medicine S233 Volume 58, N 4 S :October 2011

30.- B. H. Rowe, X. Guo, C. Villa-Roel et al. The role of triage liaison physicians on mitigating overcrowding in emergency departments: a systematic review. Academic Emergency Medicine, vol. 18, no. 2, pp. $111-120,2011$

31.-Rainer TH, Man C Senior Medical Presence at Triage Improves Service Time in Emergency Departments. Annals of Emergency Medicine Volume 51, N 4 : April 2008 pp 493

32.-Grossmann F, Zumbrunn Th Frauchiger A, Delport K, Bingisser R, Nickel Ch., At Risk of Undertriage? Testing the Performance and Accuracy of the Emergency Severity Index in Older Emergency Department Patients Ann Emerg Med. 2012;60:317-325.
33.- Abdulwahid MA, Booth A, Kuczawski M, Mason SM. The impact of senior doctor assessment at triage on emergency department performance measures: systematic review and meta-analysis of comparative studies. Emerg Med J. 2016; 33:504-513. doi:10.1136/ emermed-2014-204388

34.-Mendoza Camargo, G. Elguero Pineda, E. Sensibilidad del triage clínico en el Servicio de Urgencias Adultos del HRLALM del ISSSTE Archivos de Medicina de Urgencia de México 2011;3 (3): 93-98

35.-Baumann Michael R., Strout Tania D., Triage of Geriatric Patients in the Emergency Department: Validity and Survival With the Emergency Severity Index Ann Emerg Med. 2007;49:234-240

36.-Martínez-Segura $E$, Lleixà-Fortuño $M$, Salvadó-Usach T, Solà-Miravete $E$, Adell-Lleixà $M$, Chanovas-Borrás $M$, et al. Perfil competencial en los profesionales de triaje de los servicios de urgencias hospitalarios. Emergencias 2017;29:173-177

37.- Peng L, Hammad K. Current status of emergency department triage in mainland China: a narrative review of the literature. Nurs Health Sci 2015;17(2):148-58.

38.- Holtermann, Keith - ed. Desarrollo de sistemas de servicios de emergencias medicas: experiencia de los Estados Unidos de América para países en desarrollo. Washington, D.C: OPS, @ 2003. ISBN 9275324611

39.- Grossmann F Christ M, Winter D, Bingisser R, Platz E. Modern triage in the emergency department. Dtsch Arztebl Int 2010; 107(50): 892-8.

40.- Vásquez-Alva $\mathrm{R}$ et al Triage Hospitalario en los hospitales de Lima 2016. Informe preliminar. En prensa.
Consulte la Versión Electrónica de la Revista:

Facultad de Medicina Humana Universidad Ricardo Palma

http://revistas.urp.edu.pe/index.php/RFMH<smiles>[C]1CC2CCC1C2</smiles> 